\title{
Electroacupuncture exerts anti-inflammatory effects in cerebral ischemia-reperfusion injured rats via suppression of the TLR4/NF-kB pathway
}

\author{
LAN LAN $^{1 *}$, JING TAO $^{1 *}$, AZHEN CHEN $^{2}$, GUANLI XIE $^{3}$, JIA HUANG $^{1}$, \\ JIUMAO LIN $^{4}$, JUN PENG $^{4}$ and LIDIAN CHEN ${ }^{1}$ \\ ${ }^{1}$ College of Rehabilitation Medicine, ${ }^{2}$ MOE Key Laboratory of Traditional Chinese Medicine on Osteology and Traumatology \\ and Exercise Rehabilitation, ${ }^{3}$ Fujian Key Laboratory of Exercise Rehabilitation and ${ }^{4}$ Academy of Integrative Medicine, \\ Fujian University of Traditional Chinese Medicine, Fuzhou, Fujian 350122, P.R. China
}

Received July 31, 2012; Accepted October 1, 2012

DOI: $10.3892 / \mathrm{ijmm} .2012 .1184$

\begin{abstract}
Inflammatory response has been shown to play a critical role in brain damage after cerebral ischemia-reperfusion (I/R) injury, which is tightly regulated by the Toll-like receptor (TLR)4/nuclear factor (NF)- $\kappa \mathrm{B}$ pathway; therefore, suppression of TLR4/NF- $\kappa \mathrm{B}$ signaling has become a promising target for the anti-inflammatory treatment in ischemic stroke. Acupuncture has been used as a complementary and alternative therapy practice that supplements conventional medicine. Numerous studies have demonstrated the clinical efficacy of acupuncture in stroke rehabilitation. However, the precise mechanism of its neuroprotective effect remains poorly understood. Using a focal cerebral I/R injured rat model, in the present study we evaluated the neuroprotective and anti-inflammatory activities of electroacupuncture at Quchi and Zusanli, and investigated the underlying molecular mechanisms. We found that electroacupuncture at Quchi (LI11) and Zusanli (ST36) acupoints significantly improved the ischemia-associated scores of neurological deficits, reduced cerebral infarction and alleviated inflammatory responses. Moreover, the crucial signaling molecules in the TLR4/NF- $\kappa$ B signaling pathway were regulated by acupuncture, which coincided with suppressed secretion levels of inflammatory cytokines such as TNF- $\alpha$, IL-1 $\beta$ and IL-6. Our
\end{abstract}

Correspondence to: Dr Lidian Chen, College of Rehabilitation Medicine, Fujian University of Traditional Chinese Medicine, 1 Huatuo Road, Minhou Shangjie, Fuzhou, Fujian 350122, P.R. China E-mail: lidianchen87@yahoo.com

*Contributed equally

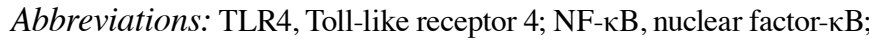
$\mathrm{I} / \mathrm{R}$, ischemia/reperfusion; MCAO, middle cerebral artery occlusion; EA, electroacupuncture; TTC, 2,3,5-triphenyltetrazolium chloride; $\mathrm{H} \& \mathrm{E}$, hematoxylin and eosin

Key words: electroacupuncture, Zusanli (ST36) and Quchi (LI11) acupoints, cerebral ischemia/reperfusion, Toll-like receptor 4/nuclear factor- $\kappa \mathrm{B}$ pathway, inflammatory response data suggest that electroacupuncture exerts a neuroprotective function in ischemic stroke through inhibition of TLR4/ $\mathrm{NF}-\kappa \mathrm{B}-$ mediated inflammation.

\section{Introduction}

Ischemic stroke is one of the most common causes of mortality and complex disability in adults worldwide (1-3). Despite improvements in the interventional techniques and pharmacological agents, there are currently no exact effective drugs and techniques to manage post-stroke rehabilitation or recovery. Acupuncture originated in ancient China and is among the oldest healing methodologies in the world. As part of traditional Chinese medicine (TCM), acupuncture has been used for thousands of years as a complementary and alternative therapy practice that supplements conventional medicine in many oriental countries as well as recently in Western society (4). A large number of studies have demonstrated the clinical efficacy of acupuncture in stroke rehabilitation (5-9). Recently, we reported that electroacupuncture alleviates neurological deficits possibly by promoting the proliferation and differentiation of nerve stem cells (10). Additionally, based on a number of documents and materials, the Zusanli (ST36) and Quchi (LI11) acupoints were commonly used in China to clinically treat stroke $(11,12)$. However, the precise mechanism of its neuroprotective effect remains largely unknown. Inflammatory response has been shown to play a critical role in brain damage after cerebral ischemia-reperfusion (I/R) injury $(13,14)$, which is tightly regulated by Toll-like receptors (TLRs). The transmembrane TLRs are a family of patternrecognition receptors (PRRs) which enable the innate and adaptive immune systems to recognize pathogen-associated molecular patterns (PAMPs). TLRs contain an intracellular Toll-interleukin 1 receptor (TIR) domain and exert their functions by interacting with TIR domain-containing adaptor proteins such as MyD88, Mal, TRIF and TRAM $(15,16)$. To date, over 13 members of the TLR family have been identified in mammals (17), of which TLR4 is the best studied. TLR4 recognizes LPS from Gram-negative bacteria and various host-derived molecules, such as heat-shock proteins, 
fibronectin, hyaluronic acid and heparan sulfate (18-20). Upon ligand binding, TLR4 undergoes a conformational change and dimerizes, which then recruits TIR domain-containing adaptor proteins, transducing the immune-related signals to the nucleus via transcription factors such as nuclear factor- $\kappa \mathrm{B}$ $(\mathrm{NF}-\kappa \mathrm{B})$. As one of the most important nuclear transcription factors, $N F-\kappa B$ is involved in the control of many critical physiological processes, such as cell proliferation, apoptosis, and especially in pathways of the immune and inflammatory responses. In unstimulated cells, $\mathrm{NF}-\kappa \mathrm{B}$ is sequestered in the cytosol via interaction with inhibitory $\mathrm{I} \kappa \mathrm{B}$ proteins. However, when cells receive pathological stimuli, $\mathrm{I} \kappa \mathrm{B}$ proteins could be phosphorylated by $\mathrm{I} \kappa \mathrm{B}$ kinase (IKK) that is activated by various mechanisms including TLR4-mediated immune signaling. Phosphorylation of I $\mathrm{B}$ proteins results in their ubiquitination and degradation, which in turn releases sequestered NF- $\kappa \mathrm{B}$, leading to its translocation to the nucleus where it induces the expression of various pro-inflammatory cytokines, such as TNF- $\alpha$, IL- $1 \beta$ and IL-6. Therefore, suppression of the TLR4/NF- $\kappa$ B pathway has become a promising target for the anti-inflammatory treatment in ischemic stroke.

Using a focal cerebral I/R injured rat model, in the present study we evaluated the neuroprotective and anti-inflammatory activities of electroacupuncture at Quchi and Zusanli, and investigated the underlying molecular mechanisms.

\section{Materials and methods}

Materials and reagents. TRIzol reagent was purchased from

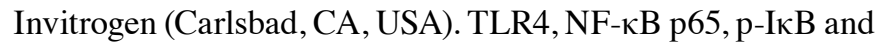
$\beta$-actin antibodies, horseradish peroxidase (HRP)-conjugated secondary antibodies were obtained from Cell Signaling Technology (Beverly, MA, USA). Rat TNF- $\alpha$, IL-1 and IL-6 ELISA kits were purchased from Shanghai XiTang Biological Technology Co., Ltd. (Shanghai, China). All the other chemicals used, unless otherwise stated, were obtained from Sigma Chemicals (St. Louis, MO, USA).

Animals. Adult male Sprague-Dawley rats (with an initial body weight of 200-250 g) were obtained from Shanghai SLAC Laboratory Animal Co., Ltd. (Shanghai, China) and housed in a temperature- and humidity-controlled room with a 12-h light/dark cycle and free access to food and water. All animal procedures were strictly in accordance with international ethical guidelines and the National Institutes of Health Guide concerning the Care and Use of Laboratory Animals, and the experiments were approved by the Institutional Animal Care and Use Committee of Fujian University of Traditional Chinese Medicine.

Establishment of the cerebral I/R injured rat model and animal grouping. The I/R injured model was generated by occlusion of the middle cerebral artery (MCA). Prior to surgery, rats were allowed access to water but fasted for $24 \mathrm{~h}$ and the surgical procedures were performed as previously described by Longa et al (21) with slight modifications. Briefly, after a rat was anesthetized with $10 \%$ chloral hydrate by intraperitoneal injection $(300 \mathrm{mg} / \mathrm{kg})$, the left common carotid artery (CCA), the left external carotid artery (ECA) and the internal carotid artery (ICA) were exposed and isolated through a midline cervical incision. An embolus was advanced through the ICA to the MCA until mild resistance was encountered $(20 \pm 2 \mathrm{~mm})$, thereby occluding the origin of the MCA. The cervical incision was closed with a silk suture. Reperfusion was achieved by withdrawing the intraluminal occlusive embolus to restore blood supply to the MCA area after $2 \mathrm{~h}$ of ischemia. For the rats in the sham group, the left CCA, ECA and ICA were exposed, but no ligations and occlusions were performed. The rectal temperature of rats was maintained at $37^{\circ} \mathrm{C}$ throughout the surgical procedures.

Animals were randomly divided into three groups $(n=8)$ : the sham operation control group (SC), the ischemia control group (IC) and the electroacupuncture group (EA). All rats were studied for behavioral parameters at 2 and $24 \mathrm{~h}$ after I/R, and then sacrificed for subsequent experiments.

EA stimulation. EA stimulation was applied to the acupuncture points of Quchi (LI11) and Zusanli (ST36) on the right paralyzed limb by using an EA stimulator instrument (Model G6805; SMIF, Shanghai, China) after recovery from operation ( $2 \mathrm{~h}$ after I/R treatment). Two stainless steel acupuncture needles $0.3 \mathrm{~mm}$ in diameter, which were connected with the output terminals, were inserted at a depth of $2-3 \mathrm{~mm}$ into the above-mentioned acupuncture points. The stimulation with 1- or $20-\mathrm{Hz}$ frequencies was generated at an intensity of the muscle twitch threshold (the muscle twitch threshold was approximately $0.01 \mathrm{~mA}$ ).

Neurological assessment. Neurological function was evaluated at 2 and $24 \mathrm{~h}$ after $\mathrm{I} / \mathrm{R}$ in a blinded fashion according to a standard scoring system on a four-point scale (21): score 0 , no apparent deficits; score 1, failure to extend the right forepaw fully; score 2, circling to the right; score 3, falling to the right; score 4 , loss of walking. In brief, rats scoring 1-3 points indicated successful model establishment.

Measurement of the infarct volumes. Following cerebral I/R injury for $24 \mathrm{~h}$, rats were euthanized under deep anesthesia using $10 \%$ chloral hydrate and perfused transcardiacally with $0.9 \% \mathrm{NaCl}$. The brains of all rats were quickly removed for dissecting into five coronal blocks at a thickness of $2 \mathrm{~mm} /$ section. The fresh slices were incubated in 2\% 2,3,5-triphenyltetrazolium chloride (Sigma, St. Louis, MO, USA) solution in phosphate-buffered saline (PBS) (Hyclone, Beijing, China) at $37^{\circ} \mathrm{C}$ for $20 \mathrm{~min}$. The normal area of brain was stained dark red based on intact mitochondrial function, whereas the infarct area remained unstained. Stained slices were photographed by a high-resolution digital camera (Cannon sx20). Using a computerized image analysis system (Motic Med 6.0 System), the total volume of infarction was determined by integration of the areas from the sections.

Hematoxylin and eosin $(H \& E)$ staining of the brain. Rats were anesthetized and perfused transcardiacally with $0.9 \% \mathrm{NaCl}$ and $4 \%$ paraformaldehyde through the left ventricle. After the brain was removed, it was fixed in cold $4 \%$ paraformaldehyde at $4^{\circ} \mathrm{C}$ for $24 \mathrm{~h}$. After dehydration, the specimens were embedded in paraffin, cut into $5-\mu \mathrm{m}$ sections and stained with H\&E. The histological morphology of each brain specimen was examined under optical microscopy. 
Direct immunofluorescence analysis of $N F-\kappa B$ p65 nuclear translocation. The paraffin sections of brain tissues were treated with microwave heat-induced epitope retrieval. After the specimens were washed three times in PBS ( $\mathrm{pH} 7.4)$, they were incubated for $1 \mathrm{~h}$ at $37^{\circ} \mathrm{C}$ with a 150 dilution of rabbit anti-rat $\mathrm{NF}-\kappa \mathrm{B}$ p65 antibody (green). The wash step was repeated. Nuclei of all cells were counterstained with DAPI. After three washes with PBS, the tissues were mounted in Prolong Gold Antifade reagent. Images were collected by a confocal fluorescence microscope (Leiss LSM710) with a magnification of x200.

Western blot analysis. Total proteins were extracted from the infarct cortex and protein concentrations were determined by BCA assay. Samples, containing $50 \mu \mathrm{g}$ proteins, were separated by electrophoresis on $12 \%$ SDS-polyacrylamide gels Subsequently, proteins were transferred onto PVDF membranes in a Tris-glycine transfer buffer. The membranes were blocked for $2 \mathrm{~h}$ with $5 \%$ nonfat dry milk at room temperature and detected with rabbit anti-TLR4, anti-NF- $\mathrm{B}$ p65, anti-p-IкB and anti- $\beta$-actin antibodies (at a dilution of $1: 1,000$ ) at $4{ }^{\circ} \mathrm{C}$ overnight, followed by incubation with the appropriate HRP-conjugated secondary antibody for $50 \mathrm{~min}$. The bands were visualized with enhanced chemiluminescence, and images were captured using a Bio-Image Analysis System (Bio-Rad, Hercules, CA, USA).

RNA extraction and RT-PCR. Total-RNA from cerebral tissues were isolated with the TRIzol reagent according to the manufacturer's instructions. Oligo(dT)-primed RNA $(1 \mu \mathrm{g})$ was reverse transcribed into cDNA, which was then used to determine the amount of TLR4, NF- $\mathrm{B}$ p 65 mRNA by PCR. The primer sequences and the sizes of the amplification were, for $\beta$-actin: forward, 5'-ACT GGC ATT GTG ATG GAC TC-3' and reverse, 5'-CAG CAC TGT GTT GGC ATA GA-3'; TLR4: forward, 5'-GGA CTC TGC CCT GCC ACC ATT TA-3' and reverse, 5'-CTT GTG CCCT GTG AGG TCG TTG A-3'; NFp65: forward, 5'-GTG CAG AAA GAA GAC ATTG AGG TG-3' and reverse, 5'-AGG CTA GGG TCA GCG TAT GG-3'. Samples were analyzed by gel electrophoresis (1.5\% agarose). The DNA bands were examined using a Gel Documentation system (Model Gel Doc 2000; Bio-Rad).

Determination of the serum level of TNF- $\alpha, I L-1 \beta$ and $I L-6$ by ELISA. Animal blood was obtained aseptically from abdominal aorta. Blood-containing tubes were allowed to stand at room temperature for $2 \mathrm{~h}$, and sera were obtained by centrifugation at $3,000 \mathrm{xg}$ for $20 \mathrm{~min}$ in $4^{\circ} \mathrm{C}$. The serum was assayed for levels of TNF- $\alpha$, IL-1 $\beta$ and IL- 6 by ELISA kits (Shanghai XiTang Biological Technology Co., Ltd.) according to the manufacture's instructions. Briefly, the wells were coated with $100 \mu \mathrm{l}$ capture antibody at $4^{\circ} \mathrm{C}$. After three washes, the wells were blocked with $200 \mu \mathrm{l}$ assay diluents at room temperature for $1 \mathrm{~h}$, followed by another three washes. Then, $100 \mu$ l diluted TNF- $\alpha$, IL- $1 \beta$ or IL- 6 standard and test samples were added and incubated for $1 \mathrm{~h}$ at $37^{\circ} \mathrm{C}$. After repeated washes, the substrate was added and incubated for $20 \mathrm{~min}$ at room temperature. Absorbance at $450 \mathrm{~nm}$ wavelength was measured, and protein concentration was determined by interpolation onto absorbance curves generated by recombinant TNF- $\alpha$, IL- $1 \beta$ or IL- 6 protein standards using an ELISA reader (Model ELX800; BioTek, USA).
Table I. Neurological assessment of rats.

\begin{tabular}{lcc}
\hline Group $(\mathrm{n}=8)$ & $2 \mathrm{~h}$ after IR & $24 \mathrm{~h}$ after IR \\
\hline SC & 0 & 0 \\
IC & $2.38 \pm 0.74$ & $2.13 \pm 0.83$ \\
EA & $2.50 \pm 0.53$ & $1.38 \pm 0.52^{\mathrm{a}}$ \\
\hline
\end{tabular}

SC, sham operation control; IC, ischemic control; EA, electroacupuncture. Data shown as averages $\pm \mathrm{SE}$ from 8 individual rats in each group. ${ }^{\mathrm{P}}<0.05$ vs. the IC group.

Statistical analysis. Statistical data are expressed as the means \pm SD. Statistical analysis was performed with the Student's t-test and ANOVA using the SPSS package for Windows (Version 16.0). Differences with $\mathrm{P}<0.05$ were considered statistically significant.

\section{Results}

Electroacupuncture at the acupoints of Zusanli (ST36) and Quchi (LI11) displays neuroprotective activity in I/R injured rats. The neuroprotective effect of electroacupuncture was examined by neurological assessment and evaluation of cerebral infarct volume. Rats in the IC and EA groups displayed obvious manifestation of neurological deficits and cerebral infarction, whereas rats in the SC group did not show any signs of cerebral injury ( $\mathrm{P}<0.05$ vs. the $\mathrm{SC}$ group), indicating the success of the model construction (Table I and Fig. 1). In addition, prior to electric stimulation, the rats in the EA group did not show any remarkable difference in clinical evaluation compared with the IC group rats. However, $24 \mathrm{~h}$ after electroacupuncture at the acupoints of Zusanli (ST36) and Quchi (LI11) the neurological function was improved and the cerebral infarct volumes were reduced significantly $(\mathrm{P}<0.05$ vs. the IC group), demonstrating the therapeutic efficacy of electroacupuncture against cerebral I/R injury.

Electroacupuncture alleviates cerebral inflammation in cerebral I/R injured rats. Brain damage caused by cerebral $\mathrm{I} / \mathrm{R}$ is partly attributed to secondary injury from inflammation for a short time after the onset of cerebral ischemia, therefore developing anti-inflammation therapies at the early stage of ischemia has become an attractive strategy to combat cerebral lesion. To determine the anti-inflammatory efficacy of acupuncture in ischemic cerebral tissues, the I/R-induced cerebral histopathological changes and the degree of inflammatory cell infiltration in infarct areas were observed under optical microscope after H\&E staining. As expected, no histopathological abnormalities and inflammatory cells were observed in the SC group rats. By contrast, in the infarct core zone of the IC group rats the glial and neuron cells appeared shrunken and showed condensed nuclei, which, however, was ameliorated by electroacupuncture (Fig. 2). Moreover, compared to the IC group, much fewer inflammatory cells were infiltrated into the cerebral infarct areas in the EA group, suggesting that electroacupuncture alleviates I/R-mediated cerebral inflammation. 



Figure 1. Effect of electroacupuncture at the Quchi (LI11) and Zusanli (ST36) acupoints on cerebral infarction in cerebral ischemia/reperfusion (I/R) injured rats. (A) At the end of the experiment, cerebral tissues from each group were coronally sectioned into 2-mm slices and then processed for TTC staining. The photographs are representative images captured by a high-resolution digital camera. SC, sham operation control; IC, ischemic control; EA, electroacupuncture. (B) Infarct volume was quantified with the Motic Med 6.0 System, which was represented as a percentage of the total brain volume. Data shown are averages with SE (error bars) from three individual rats in each group. $\mathrm{P}<0.05$ vs. the IC group.

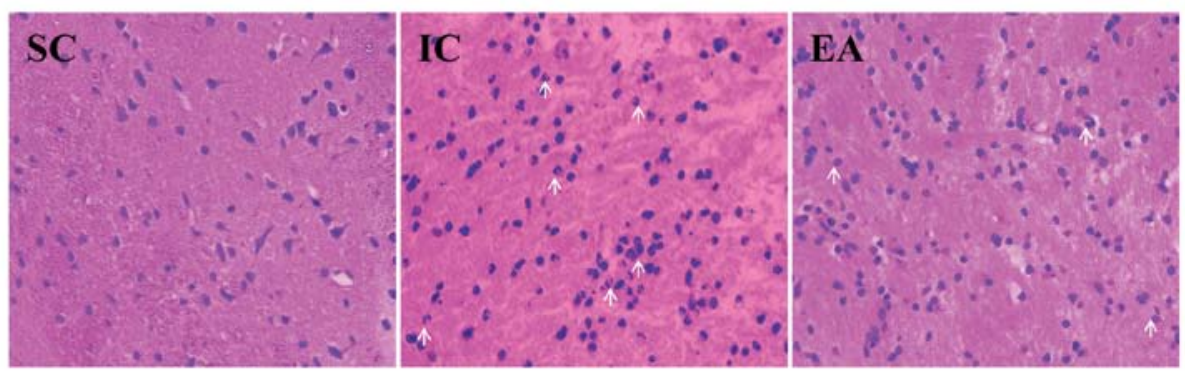

Figure 2. Effect of electroacupuncture on cerebral inflammation in cerebral $\mathrm{I} / \mathrm{R}$ injured rats. At the end of the experiment, cerebral tissues from each group ( $\mathrm{n}=5$ ) were processed for hematoxylin and eosin $(\mathrm{H} \& \mathrm{E})$ staining. I/R-induced cerebral histopathological changes and the degree of inflammatory cell infiltration in infarct areas were observed under an optical microscope at a magnification of x200. SC, sham operation control; IC, ischemic control; EA, electroacupuncture.

A

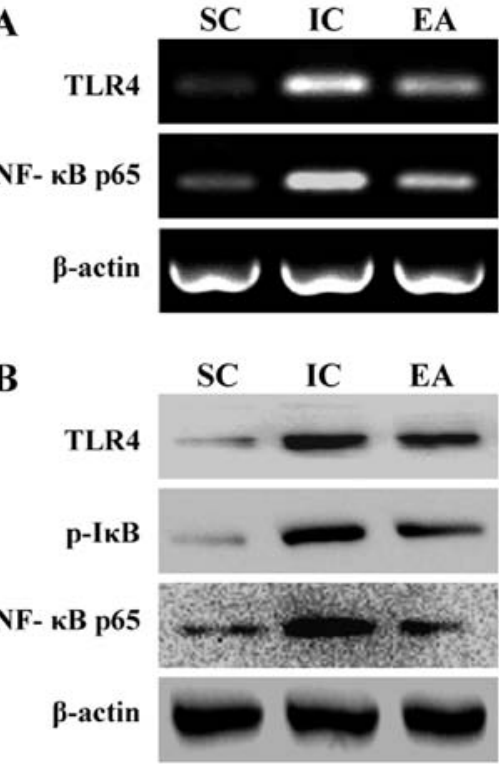

Figure 3. Effect of electroacupuncture on the TLR4/NF-кB pathway in cerebral $\mathrm{I} / \mathrm{R}$ injured rats. (A) The mRNA levels of TLR4 and NF- $\mathrm{KB}$ in ischemic cerebral tissues were determined by RT-PCR. (B) The protein expression levels of TLR4, NF- $\mathrm{KB}$ and the phosphorylation of I $\mathrm{B}$ were analyzed by western blotting. GAPDH and $\beta$-actin were used as the internal control for RT-PCR and western blotting, respectively. Data are representative of five individual rats in each group. $\mathrm{SC}$, sham operation control; IC, ischemic control; EA, electroacupuncture.
Electroacupuncture suppresses the activation of the TLR4I $N F-\kappa B$ pathway in cerebral I/R injured rats. TLR4 can activate a common signaling pathway by inducing I $\kappa \mathrm{B}$ phosphorylation and degradation and culminating the translocation of NF- $\mathrm{\kappa B}$ from cytoplasm into nucleus, which arouse downstreamassociated pro-inflammatory cytokines. To explore the anti-inflammatory mechanism of electroacupuncture, we examined its effect on the TLR4/NF- $\mathrm{KB}$ signaling pathway in ischemic cerebral tissues. The expression of TLR4 and NF- $\kappa B$ p65 as well as the phosphorylation level of I $\mathrm{KB}$ were significantly increased in the IC group compared with those in the SC group, which, however, was significantly neutralized by electroacupuncture (Fig. 3). To further verify these results, we evaluated the effect of electroacupuncture on nuclear translocation of $\mathrm{NF}-\kappa \mathrm{B}$ which is a critical step for NF- $\kappa \mathrm{B}$ activation. The NF- $\mathrm{KB}$ p65 subunit was visualized by immunofluorescence staining and the cells were counterstained with DAPI; NF- $\mathrm{KB}$ nuclear translocation was recognized by the co-localization of p65 subunit with DAPI. Cerebral I/R injury resulted in the nuclear translocation of NF- $\kappa B$ p65 subunit, which was not observed in the sham operation group (Fig. 4). However, electroacupuncture profoundly inhibited I/R-induced NF- $\mathrm{\kappa B}$ nuclear translocation. This indicates that the anti-inflammatory effect of electroacupuncture at Zusanli and Quchi is mediated by suppression of TLR4/NF- $\mathrm{KB}$ signaling in cerebral $\mathrm{I} / \mathrm{R}$ injured rats. 



Figure 4. Effect of electroacupuncture on the nuclear translocation of NF- $\kappa \mathrm{B}$ p65 subunit in cerebral I/R injured rats. At the end of the experiment, cerebral tissues were processed for immunofluorescence assay. The $\mathrm{NF}-\kappa \mathrm{B}$ p65 subunit was visualized by immunofluorescence staining (green) and the cells were counterstained with DAPI (blue). NF- $\kappa \mathrm{B}$ nuclear translocation was represented by the co-localization of p65 subunit with DAPI (indicated by white arrows). Images were captured under a confocal fluorescence microscope (Leiss LSM710) with a magnification of $\mathrm{x} 200$. Images are representative of five individual rats in each group. SC, sham operation control; IC, ischemic control; EA, electroacupuncture.

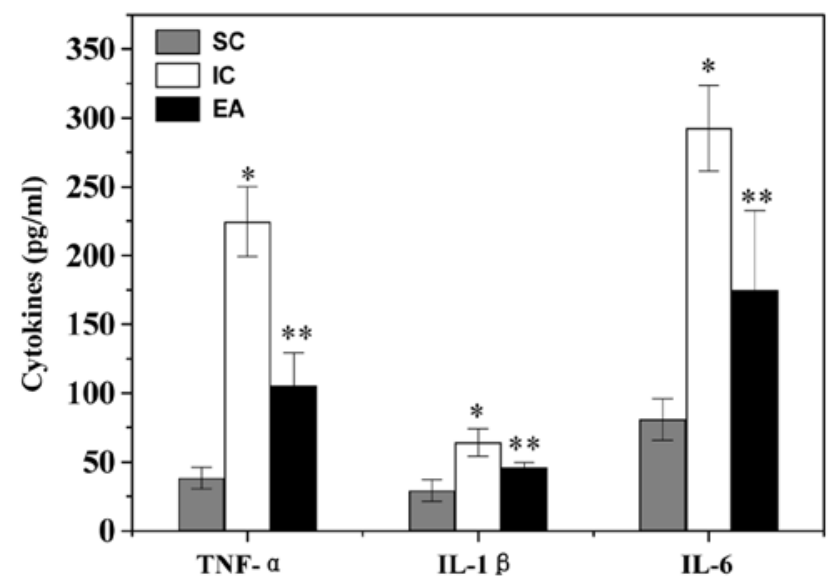

Figure 5. Effect of electroacupuncture on the secretion level of pro-inflammatory cytokines in cerebral I/R injured rats. The serum levels of TNF- $\alpha$, IL-1 $\beta$ and IL- 6 were examined by ELISA. Data are averages with SE (error bars) from five individual rats in each group. ${ }^{*} \mathrm{P}<0.05$ vs. the $\mathrm{SC}$ group; ${ }^{* *} \mathrm{P}<0.05$ vs. the IC group. SC, sham operation control; IC, ischemic control; EA, electroacupuncture.

Electroacupuncture regulates the secretion level of inflammatory cytokines in cerebral $I / R$ injured rats. To identify the downstream effectors in the TLR4/NF- $\kappa$ B pathway, the secretion level of cytokines was examined by ELISA. The serum level of pro-inflammatory TNF- $\alpha$, IL- $1 \beta$ and IL- 6 in the IC group was significantly increased, compared to that in the SC group $(\mathrm{P}<0.05)$ (Fig. 5). However, electroacupuncture at Zusanli and Quchi profoundly inhibited I/R-induced secretion of TNF- $\alpha$, IL- $1 \beta$ and IL-6 ( $<<0.05$ vs. the IC group).

\section{Discussion}

Acupuncture has served as a major complementary and alternative therapy that supplements conventional medicine and has been used in patients with acute ischemic stroke $(5-9,22)$. On the basis of a multitude of documents and materials, the Zusanli (ST36) and Quchi (LI11) acupoints were commonly used in China to clinically treat cerebral ischemia-reperfusion (I/R) injury $(11,12)$. In the present study, by using the model of MCAO followed by reperfusion which is a classical model of cerebral I/R, we demonstrated that electroacupuncture at Zusanli and Quchi displayed neuroprotective effects as evidenced by improving neurological deficits and reducing cerebral infarct volume.

Brain damage caused by cerebral I/R is partly attributed to secondary injury from inflammation for a short time after the onset of cerebral ischemia (23). Therefore, developing anti-inflammation therapies at the early stage of ischemia has been an attractive strategy to combat cerebral lesion. Acupuncture has been reported to exert anti-inflammatory effects in several non-infectious disease models of the nervous system, such as spinal cord injury and amyotrophic lateral sclerosis $(24,25)$. To determine the anti-inflammatory efficacy of acupuncture in cerebral I/R, we visualized the degree of inflammation in cerebral I/R cord by H\&E-staining. The results confirmed that electroacupuncture at Zusanli and Quchi for only $24 \mathrm{~h}$ already attenuated the histopathological changes infiltration of neutrophils in the cerebral infarct areas.

Depending on the cerebral I/R injury, TLR4 can activate a common signaling pathway by triggering I $\mathrm{B}$ phosphorylation and degradation and culminating the translocation of $\mathrm{NF}-\kappa \mathrm{B}$ from cytoplasm into nucleus, which arouse downstream-associated pro-inflammatory cytokines such as TNF- $\alpha$, IL- $1 \beta$ and IL- 6 that in turn induce inflammation response (26-28). In the present study, we found that the TLR4/NF- $\kappa$ B pathway was activated at $24 \mathrm{~h}$ after cerebral I/R injury, which was consistent with previous studies (29-31). However, the activation of TLR4/NF- $\kappa$ B signaling induced by cerebral ischemia was neutralized by systemic electroacupuncture intervention through downregulating important target genes of the TLR4/NF- $\mathrm{KB}$ pathway. As expected, we also found that electroacupuncture decreased the serum levels of pro-inflammatory cytokines (TNF- $\alpha$, IL-1 $\beta$ and IL-6).

In conclusion, we report for the first time that electroacupuncture at the Quchi (LI11) and Zusanli (ST36) acupoints on the paralyzed limb reduces ischemic brain damage, improves neurological deficits and exerts anti-inflammation function against ischemic stroke via inhibition of the TLR4/NF- $\mathrm{B}$ pathway. These results suggest that electroacupuncture may be a potential therapeutic modality for cerebral ischemia.

\section{Acknowledgements}

This study was sponsored by the Special Program for Key Basic Research Project of the China Ministry of Science and Technology (973 Program, no. 2010CB534900), and the National Natural Science Foundation of China (no. 30901935). 


\section{References}

1. Feigin VL: Stroke epidemiology in the developing world. Lancet 365: 2160-2161, 2005.

2. Carolei A, Sacco S, De Santis F and Marini C: Epidemiology of stroke. Clin Exp Hypertens 24: 479-483, 2002.

3. Jorgensen HS, Nakayama H, Pedersen PM, et al: Epidemiology of stroke-related disability. Clin Geriatr Med 15: 785-799, 1999.

4. Kim SK and Bae $\mathrm{H}$ : Acupuncture and immune modulation. Auton Neurosci 157: 38-41,2010.

5. Zhang GC, Fu WB, Xu NG, Liu JH, Zhu XP, Liang ZH, Huang YF and Chen YF: Meta analysis of the curative effect of acupuncture on post-stroke depression. J Tradit Chin Med 32: 6-11, 2012.

6. Hu HH, Chung C, Liu T, et al: A randomized controlled trial on the treatment for acute partial ischemic stroke with acupuncture. Neuroepidemiology 12: 106-113, 1993.

7. Jansen G, Lundeberg T, Kjartansson J and Samuelson U: Acupuncture and sensory neuropeptides increase cutaneous blood flow in rats. Neurosci Lett 97: 305-309, 1989.

8. Johansson K, Lindgren I, Widner H, Wiklund I and Johansson B: Can sensory stimulation improve the functional outcome in stroke patients? Neurology 43: 2189-2192, 1993.

9. Magnusson M, Johansson K and Johansson BB: Sensory stimulation promotes normalization of postural control after stroke. Stroke 25: 1176-1180, 1994

10. Tao J, Xue XH, Chen LD, Yang SL, Jiang M, Gao YL and Wang XB: Electroacupuncture improves neurological deficits and enhances proliferation and differentiation of endogenous nerve stem cells in rats with focal cerebral ischemia. Neurol Res 32: 198-204, 2010.

11. Chen W, Gu HW, Ma WP, et al: Multicentral randomized controlled study on effects of acupuncture at Zusanli (ST 36) and Xuanzhong (GB 39) on cerebrovascular function in the patient of ischemic stroke. Zhongguo Zhen Jiu 26: 851-853, 2006 (In Chinese).

12. Fu WB, Guo Y, Chen XK, et al: Comprehensive therapeutic protocol of electroacupuncture combined with Chinese herbs and rehabilitation training for treatment of cerebral infarction: a multi-center randomized controlled trial. Zhongguo Zhen Jiu 30 6-9, 2010 (In Chinese).

13. Iadecola $C$ and Alexander $M$ : Cerebral ischemia and inflammation. Curr Opin Neurol 14: 89-94, 2001.

14. Kriz J and Lalancette-Hébert M: Inflammation, plasticity and real-time imaging after cerebral ischemia. Acta Neuropathol 117: 497-509, 2009.

15. Bowie A and O'Neill L: The interleukin-1 receptor/Toll-like receptor superfamily: signal generators for pro-inflammatory interleukins and microbial products. J Leukoc Biol 67: 508-514, 2000 .
16. Slack JL, Schooley K, Bonnert TP, et al: Identification of two major sites in the type I interleukin-1 receptor cytoplasmic region responsible for coupling to pro-inflammatory signaling pathways. J Biol Chem 275: 4670-4678, 2000.

17. Kawai T and Akira S: TLR signaling. Cell Death Differ 13: $816-825,2006$

18. Johnson GB, Brunn GJ, Kodaira Y and Platt JL: Receptor-mediated monitoring of tissue well-being via detection of soluble heparan sulfate by Toll-like receptor 4. J Immunol 168: 5233-5239, 2002.

19. Lehnardt S, Schott E, Trimbuch T, et al: A vicious cycle involving release of heat shock protein 60 from injured cells and activation of Toll-like receptor 4 mediates neurodegeneration in the CNS. J Neurosci 28: 2320-2331, 2008.

20. Smiley ST, King JA and Hancock WW: Fibrinogen stimulates macrophage chemokine secretion through Toll-like receptor 4. J Immunol 167: 2887-2894, 2001.

21. Longa EZ, Weinstein PR, Carlson S and Cummins R: Reversible middle cerebral artery occlusion without craniectomy in rats. Stroke 20: 84-91, 1989.

22. Chang H, Kwon YD and Yoon SS: Use of acupuncture therapy as a supplement to conventional medical treatments for acute ischemic stroke patients in an academic medical centre in Korea. Complement Ther Med 19: 256-263, 2011.

23. Ikeda K, Negishi $\mathrm{H}$ and Yamori Y: Antioxidant nutrients and hypoxia/ischemia brain injury in rodents. Toxicology 189: 55-61, 2003.

24. Choi DC, Lee JY, Moon YJ, et al: Acupuncture-mediated inhibition of inflammation facilitates significant functional recovery after spinal cord injury. Neurobiol Dis 39: 272-282, 2010.

25. Jiang JH, Yang EJ, Baek MG, et al: Anti-inflammatory effects of electroacupuncture in the respiratory system of a symptomatic amyotrophic lateral sclerosis animal model. Neurodegener Dis 8: 504-514, 2011

26. Ridder DA and Schwaninger M: NF-kappaB signaling in cerebral ischemia. Neuroscience 158: 995-1000, 2009.

27. Barton GM and Medzhitov R: Toll-like receptor signaling pathways. Science 300: 1524-1525, 2003.

28. Blanco AM, Pascual M, Valles SL and Guerri C: Ethanolinduced iNOS and COX-2 expression in cultured astrocytes via NF-kappa B. Neuroreport 15: 681-685, 2004.

29. Gao Y, Fang X, Tong Y, et al: TLR4-mediated MyD88-dependent signaling pathway is activated by cerebral ischemia-reperfusion in cortex in mice. Biomed Pharmacother 63: 442-450, 2009.

30. Tu XK, Yang WZ, Shi SS, et al: Baicalin inhibits TLR2/4 signaling pathway in rat brain following permanent cerebral ischemia. Inflammation 34: 463-470, 2011.

31. Fan H, Li L, Zhang X, et al: Oxymatrine downregulates TLR4, TLR2, MyD88, and NF-kappaB and protects rat brains against focal ischemia. Mediators Inflamm 2009: 704706, 2009. 